\title{
The Relationship of Sodium and Potassium Conductances with Dynamic States of a Mathematical Model of Electrosensory Pyramidal Neurons
}

\author{
Takaaki Shirahata \\ Kagawa School of Pharmaceutical Sciences, Tokushima Bunri University, Sanuki, Japan \\ Email: tshi@kph.bunri-u.ac.jp
}

Received 30 March 2016; accepted 23 May 2016; published 26 May 2016

Copyright (C) 2016 by author and Scientific Research Publishing Inc. This work is licensed under the Creative Commons Attribution International License (CC BY). http://creativecommons.org/licenses/by/4.0/

(c) ()

\begin{abstract}
Electrosensory pyramidal neurons in weakly electric fish can generate burst firing. Based on the Hodgkin-Huxley scheme, a previous study has developed a mathematical model that reproduces this burst firing. This model is called the ghostbursting model and is described by a system of nonlinear ordinary differential equations. Although the dynamic state of this model is a quiescent state during low levels of electrical stimulation, an increase in the level of electrical stimulation transforms the dynamic state first into a repetitive spiking state and finally into a burst firing state. The present study performed computer simulation analysis of the ghostbursting model to evaluate the sensitivity of the three dynamic states of the model (i.e., the quiescent, repetitive spiking, and burst firing states) to variations in sodium and potassium conductance values of the model. The present numerical simulation analysis revealed the sensitivity of the electrical stimulation threshold required for eliciting the burst firing state to variations in the values of four ionic conductances (i.e., somatic sodium, dendritic sodium, somatic potassium, and dendritic potassium conductances) in the ghostbursting model.
\end{abstract}

\section{Keywords}

Mathematical Model, Computer Simulation, Ghostbursting, Ionic Conductance

\section{Introduction}

The ghostbursting model is a mathematical model of electrosensory pyramidal neurons in weakly electric fish, 
which is described by a system of nonlinear Ordinary Differential Equations (ODEs) (see Methods in [1]). This model is based on the Hodgkin-Huxley formalism and describes the time evolution of the membrane potentials of the somatic and dendritic compartments of the model. This model contains many parameters such as electrical stimulation and ionic conductances (i.e., sodium and potassium conductances), and previous studies have revealed the relationship of the dynamic states of the model with variations in parameter values. An increase in the amplitude of the electrical stimulation to the somatic compartment changes the dynamic state of the model from a quiescent state to a repetitive spiking state and finally to a bursting firing state [1]. The sensitivity of the electrical stimulation thresholds required for inducing the repetitive spiking and bursting firing states to variations in the potassium conductance of the dendritic compartment has been characterized [1]. In addition, the sensitivity of the electrical stimulation thresholds required for inducing the repetitive spiking and bursting firing states to variations in the somatic-dendritic coupling conductance has also been reported [2]. In particular, the relationship between the potassium conductance of the dendritic compartment and the dynamic states of the model has been extensively characterized; the number of spikes per burst in a two-dimensional parameter space [3] and the influence of the kinetics of the potassium conductance of the dendritic compartment on the dynamics of the model [4] have been reported. Results from these previous studies highlight the importance of extending these investigations to studies of the sensitivity of the dynamics of the ghostbursting model to parameter variations, particularly focusing on detailed analysis of the membrane conductance ([5] and page 26 in [6]).

Studies of the characteristics of the potassium conductance of the dendritic compartment have previously been carried out, as described above. However, characteristics of the potassium conductance of the somatic compartment and the sodium conductances of the somatic/dendritic compartments have not been investigated in detail. Doiron and coworkers have implied that variations in values of the sodium conductances of the somatic/ dendritic compartments or the potassium conductance of the somatic compartment may affect electrical stimulation thresholds (see the last sentence of Discussion of [1]), but this relationship has yet to be explored in detail. A systematic evaluation of the sodium and potassium conductances of both somatic and dendritic compartments is necessary for a thorough understanding of the difference between these conductances. Therefore, the present study performed computer simulation analysis of the ghostbursting model to reveal the sensitivity of the electrical stimulation thresholds to variations in the sodium and potassium conductances.

\section{Materials and Methods}

The ghostbursting model used in this study is described by a system of ODEs, which consists of six state variables: the membrane potential of the somatic compartment $\left[V_{s}(t)(\mathrm{mV})\right][t$ is time $(\mathrm{ms})]$, the activating variable of the potassium conductance of the somatic compartment $\left[n_{s}(t)\right]$, the membrane potential of the dendritic compartment $\left[V_{d}(t)(\mathrm{mV})\right]$, the inactivating variable of the sodium conductance of the dendritic compartment $\left[h_{d}(t)\right]$, the activating variable of the potassium conductance of the dendritic compartment $\left[n_{d}(t)\right]$, and the inactivating variable of the potassium conductance of the dendritic compartment $\left[p_{d}(t)\right]$. The dynamic states of the ODEs can change depending on the following system parameters: electrical stimulation of the somatic compartment $\left(I_{s}\right)$, the maximal sodium conductance of the somatic compartment $\left(g_{N a, s}\right)$, the maximal potassium conductance of the somatic compartment $\left(g_{D r, s}\right)$, the maximal sodium conductance of the dendritic compartment $\left(g_{N a, d}\right)$, and the maximal potassium conductance of the dendritic compartment $\left(g_{D r, d}\right)$. The ODEs that describe the ghostbursting model are described as follows:

$$
\begin{gathered}
\frac{\mathrm{d} V_{s}(t)}{\mathrm{d} t}=I_{s}-g_{N a, s}\left(\frac{1}{1+\mathrm{e}^{-\left(V_{s}(t)+40\right) / 3}}\right)^{2}\left(1-n_{s}(t)\right)\left(V_{s}(t)-40\right)-g_{D r, s} n_{s}(t)^{2}\left(V_{s}(t)+88.5\right) \\
-0.18\left(V_{s}(t)+70\right)-\frac{1}{0.4}\left(V_{s}(t)-V_{d}(t)\right) \\
\frac{\mathrm{d} n_{s}(t)}{\mathrm{d} t}=\frac{1}{0.39}\left(\frac{1}{\left.1+\mathrm{e}^{-\left(V_{s}(t)+40\right) / 3}-n_{s}(t)\right)}\right. \\
\frac{\mathrm{d} V_{d}(t)}{\mathrm{d} t}=-g_{N a, d}\left(\frac{1}{1+\mathrm{e}^{-\left(V_{d}(t)+40\right) / 5}}\right)^{2} h_{d}(t)\left(V_{d}(t)-40\right)-g_{D r, d} n_{d}(t)^{2} p_{d}(t)\left(V_{d}(t)+88.5\right)
\end{gathered}
$$




$$
\begin{gathered}
-0.18\left(V_{d}(t)+70\right)-\frac{1}{0.6}\left(V_{d}(t)-V_{s}(t)\right) \\
\frac{\mathrm{d} h_{d}(t)}{\mathrm{d} t}=\frac{1}{1+\mathrm{e}^{\left(V_{d}(t)+52\right) / 5}-h_{d}(t)} \\
\frac{\mathrm{d} n_{d}(t)}{\mathrm{d} t}=\frac{1}{0.9}\left(\frac{1}{1+\mathrm{e}^{-\left(V_{d}(t)+40\right) / 5}}-n_{d}(t)\right) \\
\frac{\mathrm{d} p_{d}(t)}{\mathrm{d} t}=\frac{1}{5.0}\left(\frac{1}{1+\mathrm{e}^{\left(V_{d}(t)+65\right) / 6}}-p_{d}(t)\right)
\end{gathered}
$$

$I_{s}\left(\mu \mathrm{A} / \mathrm{cm}^{2}\right)$ is changed from 5.6 to 9.6. The default values of conductances are $g_{N a, s}=55 \mathrm{mS} / \mathrm{cm}^{2}, g_{D r, s}=20$ $\mathrm{mS} / \mathrm{cm}^{2}, g_{N a, d}=5 \mathrm{mS} / \mathrm{cm}^{2}$, and $g_{D r, d}=15 \mathrm{mS} / \mathrm{cm}^{2}$. Detailed explanations of the ODEs are described in [1].

The free and open source software Scilab (http://www.scilab.org/) was used to numerically solve the ODEs (initial conditions: $V_{s}=-70 \mathrm{mV}, n_{s}=0.00005, V_{d}=-70 \mathrm{mV}, h_{d}=0.973, n_{d}=0.002$, and $p_{d}=0.697$ ). Solving the equations was performed using the lsoda solver implemented in the program Scilab.

\section{Results}

Under conditions in which all conductance values were default values [see the columns of $100 \%$ conductance in Figures 1(a)-(d)], the dynamic states of the ghostbursting model were a quiescent state ( $\times$ in Figure 1 ) when $I_{s}$

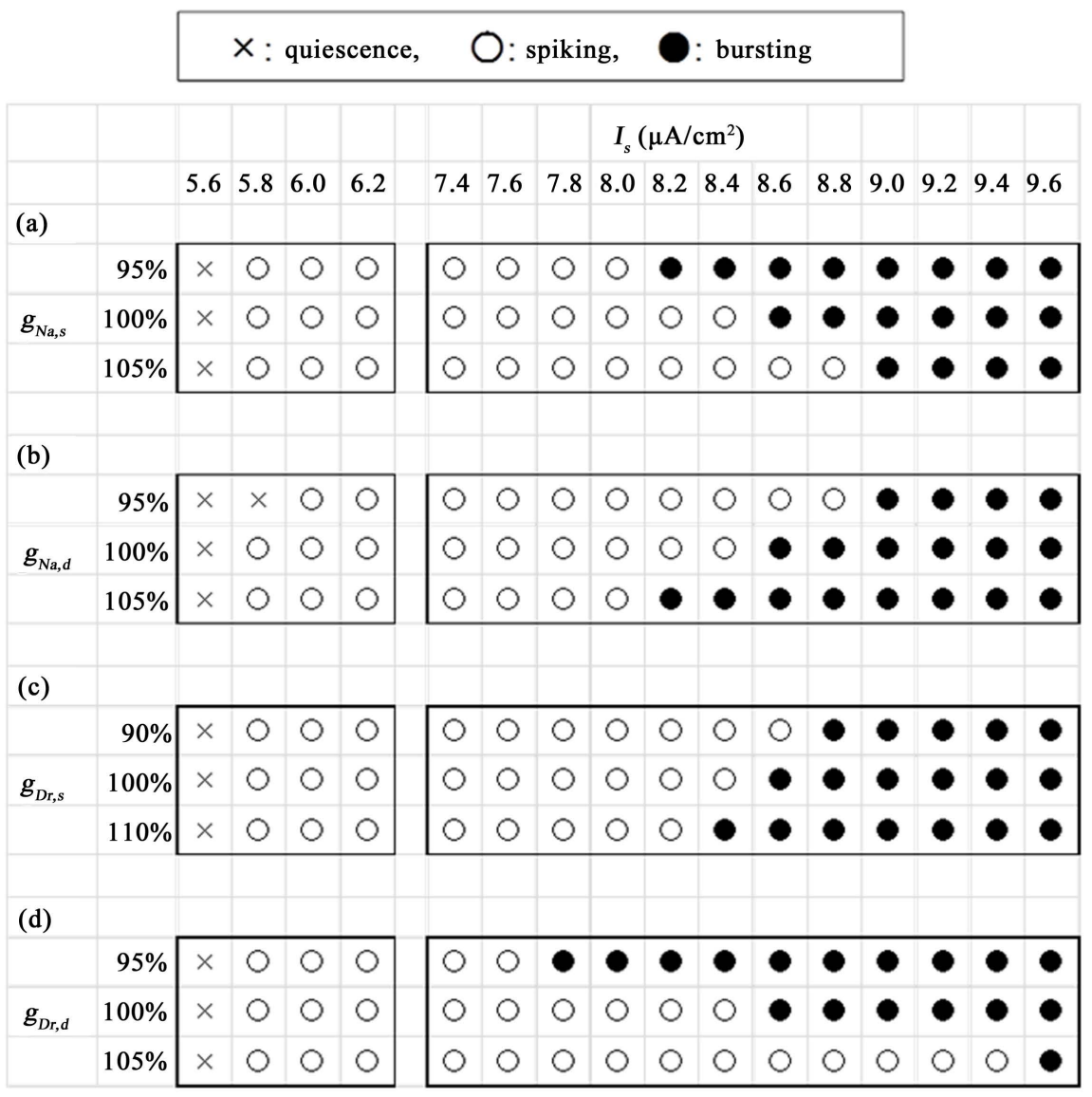

Figure 1. The dependence of the dynamic states of the ghostbursting model on various parameters. The sensitivity of the dynamic states to variations in (a) $I_{s}$ and $g_{N a, s}$, (b) $I_{s}$ and $g_{N a, d}$, (c) $I_{s}$ and $g_{D r, s}$, (d) $I_{s}$ and $g_{D r, d} \times$, quiescent state; $\circ$, repetitive spiking state; $\bullet$, bursting state. 
was 5.6, a repetitive spiking state ( $\circ$ in Figure 1 ) when $I_{s}$ was between 5.8 and 8.4, and a bursting firing state (• in Figure 1) when $I_{s}$ was between 8.6 and 9.6. The repetitive spiking threshold $\left(I_{s}=5.8\right)$ did not change even if $g_{N a, s}$ decreased to $95 \%$ or increased to $105 \%$ of the default value [Figure $1($ a) $)$. The bursting threshold $\left(I_{s}=8.6\right)$ was sensitive to variations in $g_{N a, s}$ : it decreased to 8.2 when $g_{N a, s}$ decreased to $95 \%$ of the default value, whereas it increased to 9.0 when $g_{N a, s}$ increased to $105 \%$ of the default value [Figure 1(a)]. The repetitive spiking threshold $\left(I_{s}=5.8\right)$ increased to 6.0 when $g_{N a, d}$ decreased to $95 \%$ but did not change when $g_{N a, d}$ increased to $105 \%$ [Figure 1(b)]. The bursting threshold $\left(I_{s}=8.6\right)$ was sensitive to variations in $g_{N a, d}$; it increased to 9.0 when $g_{N a, d}$ decreased to $95 \%$, whereas it decreased to 8.2 when $g_{N a, d}$ increased to $105 \%$ [Figure 1 (b)]. The repetitive spiking threshold $\left(I_{s}=5.8\right)$ did not change even if $g_{D r, s}$ decreased to $90 \%$ or increased to $110 \%$ [Figure $1(\mathrm{c})$ ]. The bursting threshold $\left(I_{s}=8.6\right)$ was sensitive to variations in $g_{D r, s}$; it increased to 8.8 when $g_{D r, s}$ decreased to $90 \%$, whereas it decreased to 8.4 when $g_{D r, s}$ increased to $110 \%$ [Figure 1 (c)]. The repetitive spiking threshold $\left(I_{s}=5.8\right)$ did not change even if $g_{D r, d}$ decreased to $95 \%$ or increased to $105 \%$ [Figure $1(\mathrm{~d})$ ]. The bursting threshold $\left(I_{s}=\right.$ 8.6) was sensitive to variations in $g_{D r, d}$; it decreased to 7.8 when $g_{D r, d}$ decreased to $95 \%$, whereas it increased to 9.6 when $g_{D r, d}$ increased to $105 \%$ [Figure $1(\mathrm{~d})$ ].

\section{Discussion}

The present numerical simulation analysis revealed the sensitivity of the repetitive spiking threshold and the bursting threshold to variations in the values of four ionic conductances (i.e., $g_{N a, s}, g_{N a, d}, g_{D r, s}$, and $g_{D r, d}$ ) in the ghostbursting model. A previous study illustrated the sensitivity of the repetitive spiking threshold and the bursting threshold to variations in $g_{D r, d}$ [1]. In addition, the same study implied that these thresholds are also sensitive to variations in other ionic conductances such as $g_{N a, s}, g_{N a, d}$, and $g_{D r, s}$. However, this was not shown explicitly [1].

The importance of the present findings are that they clearly demonstrate the sensitivity of the repetitive spiking threshold and the bursting threshold to variations in $g_{N a, s}, g_{N a, d}$, and $g_{D r, s}$. Specifically, 1) similar to the case of $g_{D r, d}$, the repetitive spiking threshold is insensitive to variations in the other ionic conductances, except $g_{N a, d}$; 2) the bursting threshold is sensitive to variations in the other three ionic conductances; 3 ) similar to the previous case, in which an increase in $g_{D r, d}$ increases the bursting threshold [1], an increase in $g_{N a, s}$ increases the bursting threshold, whereas an increase in $g_{N a, d}$ and $g_{D r, s}$ decreases the bursting threshold; and 4) the degree of sensitivity occurs as follows: $g_{D r, d}>g_{N a, s}=g_{N a, d}>g_{D r, s}$.

The effect of variations in ionic conductance values on the behaviors of mathematical models of excitable cells is an important topic of investigation. For example, action potential duration is differentially modulated by variations in the slow-inward calcium conductance $\left(G_{s i}\right)$ versus the delayed rectifier potassium conductance $\left(G_{K}\right)$ in the LR1 model [7]. Similarly, the distinct roles of several ionic conductances $\left(G_{C a S}, G_{h}, G_{K d}, G_{A}\right.$, and $\left.G_{K C a}\right)$ in regulating maximal gain modulation is revealed in a model of lobster somatogastric neurons [8]. The present study advances our understanding of membrane dynamics in pyramidal neurons by revealing differences in bursting threshold changes among $g_{N a, s}, g_{N a, d}, g_{D r, s}$, and $g_{D r, d}$. Notably, the present study examines conductances of the same ion type in both somatic and dendritic compartments (i.e., the model incorporates differences between $g_{N a, s}$ and $g_{N a, d}$, and between $g_{D r, s}$ and $g_{D r, d}$ ). Previous studies described above [7] [8] investigated a singlecompartment conductance-based model, and thus, could not reveal the differences in ionic conductances between different compartments.

\section{Conclusion}

Previous work has reported the sensitivity of the repetitive spiking threshold and the bursting threshold to variations in $g_{D r, d}$ but has not characterized the sensitivity of these thresholds to variations in three other ionic conductances $\left(g_{N a, s}, g_{N a, d}\right.$, and $\left.g_{D r, s}\right)$ [1]. The present study addresses this issue and contributes to a thorough understanding of differential modulation of these thresholds by variations in the four ionic conductances (i.e., $g_{N a, s}$, $g_{N a, d}, g_{D r, s}$, and $\left.g_{D r, d}\right)$ in the ghostbursting model.

\section{Acknowledgements}

The author would like to thank Enago (www.enago.jp) for the English language review. 


\section{References}

[1] Doiron, B., Laing, C., Longtin, A. and Maler, L. (2002) Ghostbursting: A Novel Neuronal Burst Mechanism. Journal of Computational Neuroscience, 12, 5-25. http://dx.doi.org/10.1023/A:1014921628797

[2] Laing, C., Doiron, B., Longtin, A. and Maler, L. (2002) Ghostbursting: The Effects of Dendrites on Spike Patterns. Neurocomputing, 44-46, 127-132. http://dx.doi.org/10.1016/S0925-2312(02)00373-9

[3] Shirahata, T. (2012) Analysis of the Electrosensory Pyramidal Cell Bursting Model for Weakly Electric Fish: Model Prediction under Low Levels of Dendritic Potassium Conductance. Acta Biologica Hungarica, 63, 313-320. http://dx.doi.org/10.1556/ABiol.63.2012.3.1

[4] Shirahata, T. (2015) Evaluation of Kinetic Properties of Dendritic Potassium Current in Ghostbursting Model of Electrosensory Neurons. Applied Mathematics, 6, 128-135. http://dx.doi.org/10.4236/am.2015.61013

[5] Shirahata, T. (2015) Numerical Study of a Mathematical Model of Vibrissa Motoneurons: The Relationship between Repetitive Spiking and Two Types of Sodium Conductance. International Journal of Theoretical and Mathematical Physics, 5, 48-52.

[6] Ashrafuzzaman, M. and Tuszynski, J. (2012) Membrane Biophysics. Springer, Berlin.

[7] Sobie, E.A. (2009) Parameter Sensitivity Analysis in Electrophysiological Models Using Multivariable Regression. Biophysical Journal, 96, 1264-1274. http://dx.doi.org/10.1016/j.bpj.2008.10.056

[8] Patel, A.X. and Burdakov, D. (2015) Mechanisms of Gain Control by Voltage-Gated Channels in Intrinsically-Firing Neurons. PLoS ONE, 10, e0115431. http://dx.doi.org/10.1371/journal.pone.0115431 